ARTICLE

\title{
Structural basis for Mep2 ammonium transceptor activation by phosphorylation
}

Bert van den Berg ${ }^{1}$, Anupama Chembath ${ }^{1}$, Damien Jefferies ${ }^{2}$, Arnaud Basle $^{1}$, Syma Khalid $^{2}$ \& Julian C. Rutherford ${ }^{1}$

Mep2 proteins are fungal transceptors that play an important role as ammonium sensors in fungal development. Mep2 activity is tightly regulated by phosphorylation, but how this is achieved at the molecular level is not clear. Here we report X-ray crystal structures of the Mep2 orthologues from Saccharomyces cerevisiae and Candida albicans and show that under nitrogen-sufficient conditions the transporters are not phosphorylated and present in closed, inactive conformations. Relative to the open bacterial ammonium transporters, non-phosphorylated Mep2 exhibits shifts in cytoplasmic loops and the C-terminal region (CTR) to occlude the cytoplasmic exit of the channel and to interact with His2 of the twin-His motif. The phosphorylation site in the CTR is solvent accessible and located in a negatively charged pocket $\sim 30 \AA$ away from the channel exit. The crystal structure of phosphorylationmimicking Mep2 variants from $C$. albicans show large conformational changes in a conserved and functionally important region of the CTR. The results allow us to propose a model for regulation of eukaryotic ammonium transport by phosphorylation.

\footnotetext{
${ }^{1}$ Institute for Cell and Molecular Biosciences, The Medical School, Newcastle University, Newcastle upon Tyne NE2 4HH, UK. ${ }^{2}$ School of Chemistry, University of Southampton, Highfield Campus, Southampton SO17 1BJ, UK. Correspondence and requests for materials should be addressed to B.v.d.B. (email: bert.van-den-berg@ncl.ac.uk).
} 
$\mathrm{T}$ ransceptors are membrane proteins that function not only as transporters but also as receptors/sensors during nutrient sensing to activate downstream signalling pathways $^{1}$. A common feature of transceptors is that they are induced when cells are starved for their substrate. While most studies have focused on the Saccharomyces cerevisiae transceptors for phosphate (Pho84), amino acids (Gap1) and ammonium (Mep2), transceptors are found in higher eukaryotes as well (for example, the mammalian SNAT2 amino-acid transporter and the GLUT2 glucose transporter) ${ }^{2}$. One of the most important unresolved questions in the field is how the transceptors couple to downstream signalling pathways. One hypothesis is that downstream signalling is dependent on a specific conformation of the transporter ${ }^{2}$.

Mep2 (methylammonium (MA) permease) proteins are ammonium transceptors that are ubiquitous in fungi. They belong to the Amt/Mep/Rh family of transporters that are present in all kingdoms of life and they take up ammonium from the extracellular environment ${ }^{3}$. Fungi typically have more than one Mep paralogue, for example, Mep1-3 in S. cerevisiae $^{4}$. Of these, only Mep2 proteins function as ammonium receptors/sensors in fungal development ${ }^{5}$. Under conditions of nitrogen limitation, Mep2 initiates a signalling cascade that results in a switch from the yeast form to filamentous (pseudohyphal) growth ${ }^{6}$ that may be required for fungal pathogenicity ${ }^{7}$. As is the case for other transceptors, it is not clear how Mep2 interacts with downstream signalling partners, but the protein kinase $\mathrm{A}$ and mitogenactivated protein kinase pathways have been proposed as downstream effectors of Mep2 (refs 6,8,9). Compared with Mep1 and Mep3, Mep2 is highly expressed and functions as a low-capacity, high-affinity transporter in the uptake of $\mathrm{MA}^{10}$. In addition, Mep2 is also important for uptake of ammonium produced by growth on other nitrogen sources ${ }^{11}$.

With the exception of the human RhCG structure $^{12}$, no structural information is available for eukaryotic ammonium transporters. By contrast, several bacterial Amt orthologues have been characterized in detail via high-resolution crystal structures and a number of molecular dynamics (MD) studies ${ }^{13-19}$. All the solved structures including that of RhCG are very similar, establishing the basic architecture of ammonium transporters. The proteins form stable trimers, with each monomer having 11 transmembrane (TM) helices and a central channel for the transport of ammonium. All structures show the transporters in open conformations. Intriguingly, fundamental questions such as the nature of the transported substrate and the transport mechanism are still controversial. Where earlier studies favoured the transport of ammonia gas ${ }^{13,20}$, recent data and theoretical considerations suggest that $\mathrm{Amt} / \mathrm{Mep}$ proteins are instead active, electrogenic transporters of either $\mathrm{NH}_{4}^{+}$(uniport) or $\mathrm{NH}_{3} / \mathrm{H}^{+}$(symport) $)^{15,17,21-24}$. A highly conserved pair of channel-lining histidine residues dubbed the twin-His motif may serve as a proton relay system while $\mathrm{NH}_{3}$ moves through the channel during $\mathrm{NH}_{3} / \mathrm{H}^{+}$symport ${ }^{16}$.

Ammonium transport is tightly regulated. In animals, this is due to toxicity of elevated intracellular ammonium levels ${ }^{25}$, whereas for microorganisms ammonium is a preferred nitrogen source. In bacteria, amt genes are present in an operon with $g \ln K$, encoding a $\mathrm{P}_{\mathrm{II}}$-like signal transduction class protein ${ }^{26}$. By binding tightly to Amt proteins without inducing a conformational change in the transporter ${ }^{27}$, GlnK sterically blocks ammonium conductance when nitrogen levels are sufficient. Under conditions of nitrogen limitation, GlnK becomes uridylated, blocking its ability to bind and inhibit Amt proteins ${ }^{28}$. Importantly, eukaryotes do not have GlnK orthologues and have a different mechanism for regulation of ammonium transport activity. In plants, transporter phosphorylation and dephosphorylation are known to regulate activity ${ }^{29}$. In S. cerevisiae, phosphorylation of Ser457 within the C-terminal region (CTR) in the cytoplasm was recently proposed to cause Mep2 opening, possibly via inducing a conformational change ${ }^{30}$.

To elucidate the mechanism of Mep2 transport regulation, we present here X-ray crystal structures of the Mep2 transceptors from S. cerevisiae and C. albicans. The structures are similar to each other but show considerable differences to all other ammonium transporter structures. The most striking difference is the fact that the Mep2 proteins have closed conformations. The putative phosphorylation site is solvent accessible and located in a negatively charged pocket $\sim 30 \AA$ away from the channel exit. The channels of phosphorylation-mimicking mutants of C. albicans Mep2 are still closed but show large conformational changes within a conserved part of the CTR. Together with a structure of a C-terminal Mep2 variant lacking the segment containing the phosphorylation site, the results allow us to propose a structural model for phosphorylation-based regulation of eukaryotic ammonium transport.

\section{Results}

General architecture of Mep2 ammonium transceptors. The Mep2 protein of S. cerevisiae (ScMep2) was overexpressed in S. cerevisiae in high yields, enabling structure determination by X-ray crystallography using data to $3.2 \AA$ resolution by molecular replacement (MR) with the archaebacterial Amt-1 structure (see Methods section). Given that the modest resolution of the structure and the limited detergent stability of ScMep2 would likely complicate structure-function studies, several other fungal Mep2 orthologues were subsequently overexpressed and screened for diffraction-quality crystals. Of these, Mep2 from C. albicans (CaMep2) showed superior stability in relatively harsh detergents such as nonyl-glucoside, allowing structure determination in two different crystal forms to high resolution (up to $1.5 \AA$ ). Despite different crystal packing (Supplementary Table 1), the two CaMep2 structures are identical to each other and very similar to ScMep2 $\left(C_{\alpha}\right.$ r.m.s.d. (root mean square deviation) $=0.7 \AA$ for 434 residues), with the main differences confined to the $\mathrm{N}$ terminus and the CTR (Fig. 1). Electron density is visible for the entire polypeptide chains, with the exception of the C-terminal 43 (ScMep2) and 25 residues (CaMep2), which are poorly conserved and presumably disordered. Both Mep2 proteins show the archetypal trimeric assemblies in which each monomer consists of 11 TM helices surrounding a central pore. Important functional features such as the extracellular ammonium binding site, the Phe gate and the twin-His motif within the hydrophobic channel are all very similar to those present in the bacterial transporters and RhCG ${ }^{12-19}$. In the remainder of the manuscript, we will specifically discuss CaMep2 due to the superior resolution of the structure. Unless specifically stated, the drawn conclusions also apply to ScMep2.

While the overall architecture of Mep2 is similar to that of the prokaryotic transporters $\left(C_{\alpha}\right.$ r.m.s.d. with Amt- $1=1.4 \AA$ for 361 residues), there are large differences within the $\mathrm{N}$ terminus, intracellular loops (ICLs) ICL1 and ICL3, and the CTR. The N termini of the Mep2 proteins are $\sim 20-25$ residues longer compared with their bacterial counterparts (Figs 1 and 2), substantially increasing the size of the extracellular domain. Moreover, the $\mathrm{N}$ terminus of one monomer interacts with the extended extracellular loop ECL5 of a neighbouring monomer. Together with additional, smaller differences in other extracellular loops, these changes generate a distinct vestibule leading to the ammonium binding site that is much more pronounced than in the bacterial proteins. The N-terminal vestibule and the resulting inter-monomer interactions likely increase the stability 
a

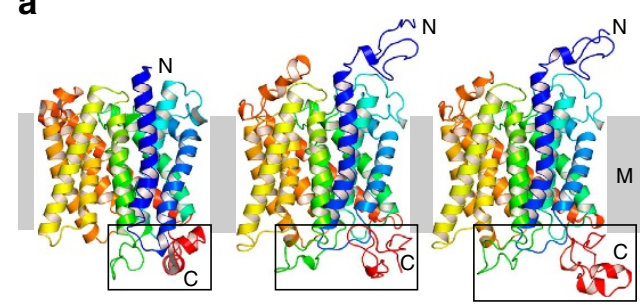

b

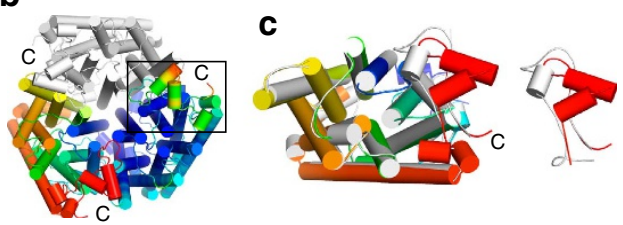

Figure 1 | X-ray crystal structures of Mep2 transceptors. (a) Monomer cartoon models viewed from the side for (left) A. fulgidus Amt-1 (PDB ID 2B2H), S. cerevisiae Mep2 (middle) and C. albicans Mep2 (right). The cartoons are in rainbow representation. The region showing ICL1 (blue), ICL3 (green) and the CTR (red) is boxed for comparison. (b) CaMep2 trimer viewed from the intracellular side (right). One monomer is coloured as in $\mathbf{a}$ and one monomer is coloured by B-factor (blue, low; red; high). The CTR is boxed. (c) Overlay of ScMep2 (grey) and CaMep2 (rainbow), illustrating the differences in the CTRs. All structure figures were generated with Pymol ${ }^{62}$.

of the Mep2 trimer, in support of data for plant AMT proteins ${ }^{31}$. However, given that an N-terminal deletion mutant (2-27 $\Delta$ ) grows as well as wild-type (WT) Mep2 on minimal ammonium medium (Fig. 3 and Supplementary Fig. 1), the importance of the $\mathrm{N}$ terminus for Mep2 activity is not clear.

Mep2 channels are closed by a two-tier channel block. The largest differences between the Mep2 structures and the other known ammonium transporter structures are located on the intracellular side of the membrane. In the vicinity of the Mep2 channel exit, the cytoplasmic end of TM2 has unwound, generating a longer ICL1 even though there are no insertions in this region compared to the bacterial proteins (Figs 2 and 4). ICL1 has also moved inwards relative to its position in the bacterial Amts. The largest backbone movements of equivalent residues within ICL1 are $\sim 10 \AA$, markedly affecting the conserved basic RxK motif (Fig. 4). The head group of Arg54 has moved $\sim 11 \AA$ relative to that in Amt-1, whereas the shift of the head group of the variable Lys 55 residue is almost $20 \AA$. The side chain of Lys56 in the basic motif points in an opposite direction in the Mep2 structures compared with that of, for example, Amt-1 (Fig. 4). In addition to changing the RxK motif, the movement of ICL1 has another, crucial functional consequence. At the C-terminal end of TM1, the side-chain hydroxyl group of the relatively conserved Tyr49 (Tyr53 in ScMep2) makes a strong hydrogen bond with the $\varepsilon_{2}$ nitrogen atom of the absolutely conserved His342 of the twin-His motif (His348 in ScMep2), closing the channel (Figs 4 and 5). In bacterial Amt proteins, this Tyr side chain is rotated $\sim 4 \AA$ away as a result of the different conformation of TM1, leaving the channel open and the histidine available for its putative role in substrate transport (Supplementary Fig. 2) ${ }^{16}$.

Compared with ICL1, the backbone conformational changes observed for the neighbouring ICL2 are smaller, but large shifts are nevertheless observed for the conserved residues Glu140 and Arg141 (Fig. 4). Finally, the important ICL3 linking the pseudosymmetrical halves (TM1-5 and TM6-10) of the transporter is also shifted up to $\sim 10 \AA$ and forms an additional barrier that closes the channel on the cytoplasmic side (Fig. 5). This two-tier channel block likely ensures that very little ammonium transport will take place under nitrogen-sufficient conditions. The closed state of the channel might also explain why no density, which could correspond to ammonium (or water), is observed in the hydrophobic part of the Mep2 channel close to the twin-His motif. Significantly, this is also true for ScMep2, which was crystallized in the presence of $0.2 \mathrm{M}$ ammonium ions (see Methods section).

The final region in Mep2 that shows large differences compared with the bacterial transporters is the CTR. In Mep2, the CTR has moved away and makes relatively few contacts with the main body of the transporter, generating a more elongated protein (Figs 1 and 4). By contrast, in the structures of bacterial proteins, the CTR is docked tightly onto the N-terminal half of the transporters (corresponding to TM1-5), resulting in a more compact structure. This is illustrated by the positions of the five universally conserved residues within the CTR, that is, Arg415(370), Glu421(376), Gly424(379), Asp426(381) and Tyr 435(390) in CaMep2(Amt-1) (Fig. 2). These residues include those of the 'ExxGxD' motif, which when mutated generate inactive transporters ${ }^{32,33}$. In Amt-1 and other bacterial ammonium transporters, these CTR residues interact with residues within the $\mathrm{N}$-terminal half of the protein. On one side, the Tyr390 hydroxyl in Amt-1 is hydrogen bonded with the side chain of the conserved His185 at the C-terminal end of loop ICL3. At the other end of ICL3, the backbone carbonyl groups of Gly172 and Lys173 are hydrogen bonded to the side chain of Arg370. Similar interactions were also modelled in the active, non-phosphorylated plant AtAmt-1;1 structure (for example, $\mathrm{Y} 467-\mathrm{H} 239$ and $\mathrm{D} 458-\mathrm{K} 71)^{33}$. The result of these interactions is that the CTR 'hugs' the N-terminal half of the transporters (Fig. 4). Also noteworthy is Asp381, the side chain of which interacts strongly with the positive dipole on the $\mathrm{N}$-terminal end of TM2. This interaction in the centre of the protein may be particularly important to stabilize the open conformations of ammonium transporters. In the Mep2 structures, none of the interactions mentioned above are present.

Phosphorylation target site is at the periphery of Mep2. Recently Boeckstaens et al. provided evidence that Ser457 in ScMep2 (corresponding to Ser453 in CaMep2) is phosphorylated by the TORC1 effector kinase Npr1 under nitrogen-limiting conditions $^{30}$. In the absence of Npr1, plasmid-encoded WT Mep2 in a $S$. cerevisiae mep1-34 strain (triple mep $\Delta$ ) does not allow growth on low concentrations of ammonium, suggesting that the transporter is inactive (Fig. 3 and Supplementary Fig. 1) s. $^{30}$. Conversely, the phosphorylation-mimicking S457D variant is active both in the triple mep $\Delta$ background and in a triple mep $\Delta$ nprld strain (Fig. 3) ${ }^{30}$. Mutation of other potential phosphorylation sites in the CTR did not support growth in the npr1s background ${ }^{30}$. Collectively, these data suggest that phosphorylation of Ser457 opens the Mep2 channel to allow ammonium uptake. Ser457 is located in a part of the CTR that is conserved in a subgroup of Mep2 proteins, but which is not present in bacterial proteins (Fig. 2). This segment (residues 450-457 in ScMep2 and 446-453 in CaMep2) was dubbed an autoinhibitory (AI) region based on the fact that its removal generates an active transporter in the absence of Npr1 (Fig. 3) ${ }^{30}$.

Where is the AI region and the Nprl phosphorylation site located? Our structures reveal that surprisingly, the AI region is folded back onto the CTR and is not located near the centre of the trimer as expected from the bacterial structures (Fig. 4). The AI region packs against the cytoplasmic ends of TM2 and TM4, 


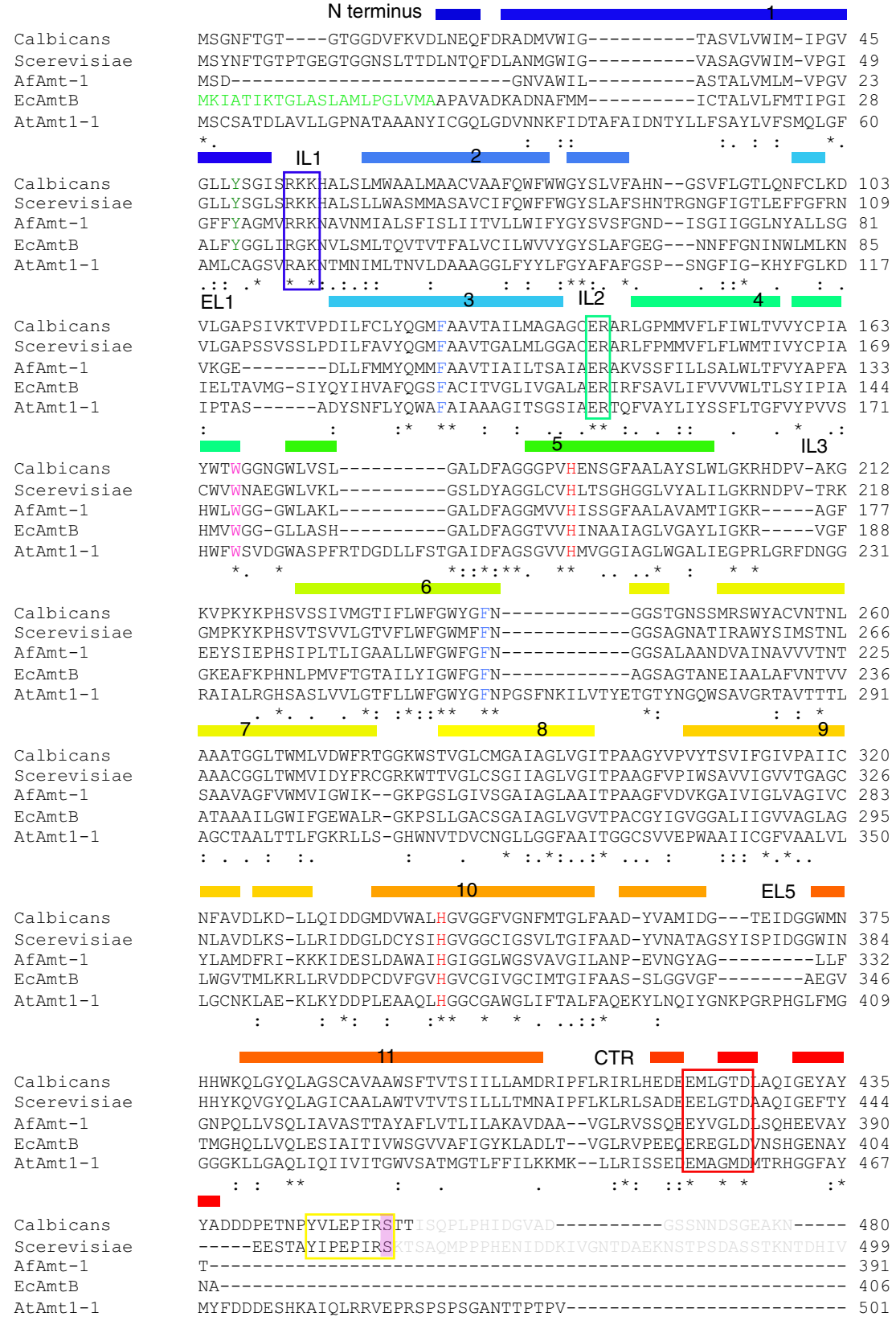

Figure 2 | Sequence conservation in ammonium transporters. ClustalW alignment of CaMep2, ScMep2, A. fulgidus Amt-1, E. coli AmtB and A. thaliana Amt-1;1. The secondary structure elements observed for CaMep2 are indicated, with the numbers corresponding to the centre of the TM segment. Important regions are labelled. The conserved RxK motif in ICL1 is boxed in blue, the ER motif in ICL2 in cyan, the conserved ExxGxD motif of the CTR in red and the Al region in yellow. Coloured residues are functionally important and correspond to those of the Phe gate (blue), the binding site Trp residue (magenta) and the twin-His motif (red). The Npr1 kinase site in the Al region is highlighted pink. The grey sequences at the $\mathrm{C}$ termini of CaMep2 and ScMep2 are not visible in the structures and are likely disordered.

physically linking the main body of the transporter with the CTR via main chain interactions and side-chain interactions of Val447, Asp449, Pro450 and Arg452 (Fig. 6). The AI regions have very similar conformations in CaMep2 and ScMep2, despite considerable differences in the rest of the CTR (Fig. 6). Strikingly, the Npr1 target serine residue is located at the periphery of the trimer, far away $(\sim 30 \AA)$ from any channel exit (Fig. 6). Despite its location at the periphery of the trimer, the electron density for the serine is well defined in both Mep2 structures and corresponds to the non-phosphorylated state (Fig. 6). This makes sense since the proteins were expressed in rich medium and confirms the recent suggestion by Boeckstaens et al. that the non-phosphorylated form of Mep2 corresponds to the inactive state $^{30}$. For ScMep2, Ser457 is the most C-terminal residue for which electron density is visible, indicating that the region beyond Ser457 is disordered. In CaMep2, the visible part of the sequence extends for two residues beyond Ser453 (Fig. 6). The peripheral location and disorder of the CTR beyond the kinase target site should facilitate the phosphorylation by Npr1. The disordered part of the CTR is not conserved in ammonium transporters (Fig. 2), suggesting that it is not important for transport. Interestingly, a ScMep2 $457 \Delta$ truncation mutant in which a His-tag directly follows Ser457 is highly expressed but has low activity (Fig. 3 and Supplementary Fig. 1b), suggesting 


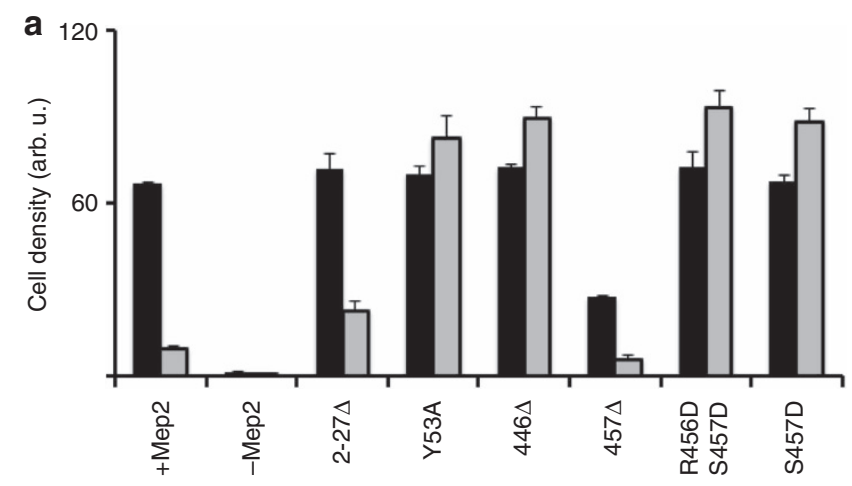

b

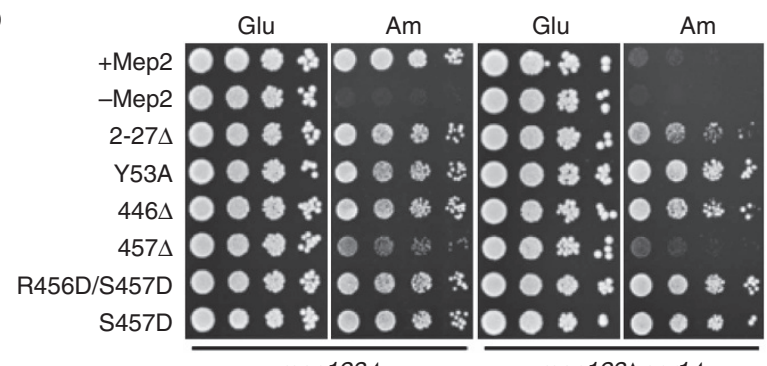

mep1234

mep123Anpr14

Figure 3 | Growth of ScMep2 variants on low ammonium medium.

(a) The triple mep $\Delta$ strain (black) and triple mep $\Delta$ npr1 $\Delta$ strain (grey) containing plasmids expressing WT and variant ScMep2 were grown on minimal medium containing $1 \mathrm{mM}$ ammonium sulphate. The quantified cell density reflects logarithmic growth after $24 \mathrm{~h}$. Error bars are the s.d. for three replicates of each strain (b) The strains used in a were also serially diluted and spotted onto minimal agar plates containing glutamate $(0.1 \%)$ or ammonium sulphate $(1 \mathrm{mM})$, and grown for 3 days at $30^{\circ} \mathrm{C}$.

that the His-tag interferes with phosphorylation by Npr1. The same mutant lacking the His-tag has WT properties (Supplementary Fig. 1b), confirming that the region following the phosphorylation site is dispensable for function.

Mep2 lacking the AI region is conformationally heterogeneous. Given that Ser457/453 is far from any channel exit (Fig. 6), the crucial question is how phosphorylation opens the Mep2 channel to generate an active transporter. Boeckstaens $e t$ al. proposed that phosphorylation does not affect channel activity directly, but instead relieves inhibition by the AI region. The data behind this hypothesis is the observation that a ScMep2 449-485 $\Delta$ deletion mutant lacking the AI region is highly active in MA uptake both in the triple mep $\Delta$ and triple mep $\Delta$ nprl$\Delta$ backgrounds, implying that this Mep2 variant has a constitutively open channe ${ }^{30}$. We obtained a similar result for ammonium uptake by the $446 \Delta$ mutant (Fig. 3), supporting the data from Marini et al. We then constructed and purified the analogous CaMep2 $442 \Delta$ truncation mutant and determined the crystal structure using data to $3.4 \AA$ resolution. The structure shows that removal of the AI region markedly increases the dynamics of the cytoplasmic parts of the transporter. This is not unexpected given the fact that the AI region bridges the CTR and the main body of Mep2 (Fig. 6). Density for ICL3 and the CTR beyond residue Arg415 is missing in the $442 \Delta$ mutant, and the density for the other ICLs including ICL1 is generally poor with visible parts of the structure having high B-factors (Fig. 7). Interestingly, however, the Tyr49-His342 hydrogen bond that closes the channel in the WT protein is still present (Fig. 7 and Supplementary Fig. 2). Why then does this mutant appear to be constitutively active? We propose two a
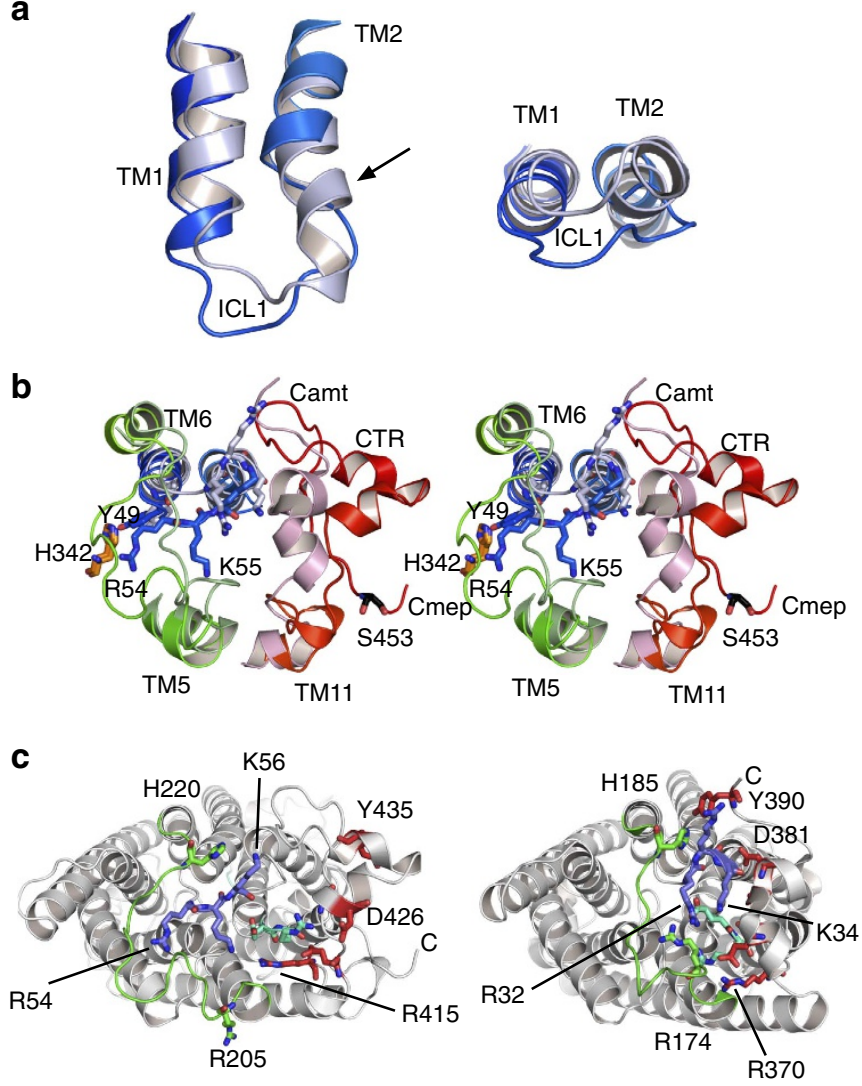

Figure 4 | Structural differences between Mep2 and bacterial ammonium transporters. (a) ICL1 in AfAmt-1 (light blue) and CaMep2 (dark blue), showing unwinding and inward movement in the fungal protein. (b) Stereo diagram viewed from the cytosol of ICL1, ICL3 (green) and the CTR (red) in AfAmt-1 (light colours) and CaMep2 (dark colours). The side chains of residues in the RxK motif as well as those of Tyr49 and His342 are labelled. The numbering is for CaMep2. (c) Conserved residues in ICL1-3 and the CTR. Views from the cytosol for CaMep2 (left) and AfAmt-1, highlighting the large differences in conformation of the conserved residues in ICL1 (RxK motif; blue), ICL2 (ER motif; cyan), ICL3 (green) and the CTR (red). The labelled residues are analogous within both structures. $\ln \mathbf{b}$ and $\mathbf{c}$, the centre of the trimer is at top.

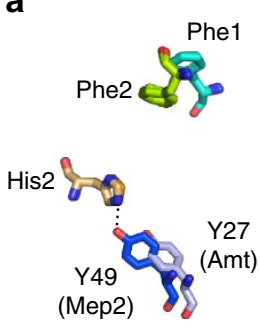

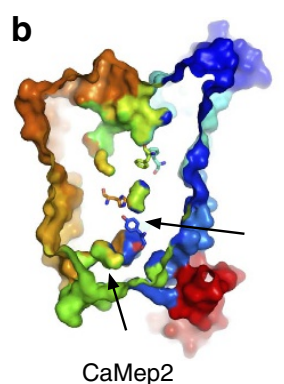

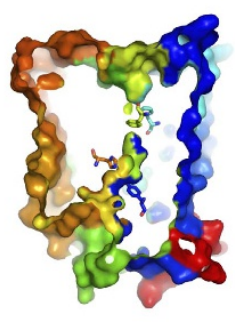

AfAmt-1
Figure 5 | Channel closures in Mep2. (a) Stereo superposition of AfAmt-1 and CaMep2 showing the residues of the Phe gate, His2 of the twin-His motif and the tyrosine residue $\mathrm{Y} 49$ in TM1 that forms a hydrogen bond with His2 in CaMep2. (b) Surface views from the side in rainbow colouring, showing the two-tier channel block (indicated by the arrows) in CaMep2.

possibilities. The first one is that the open state is disfavoured by crystallization because of lower stability or due to crystal packing constraints. The second possibility is that the Tyr-His hydrogen bond has to be disrupted by the incoming substrate to open the 


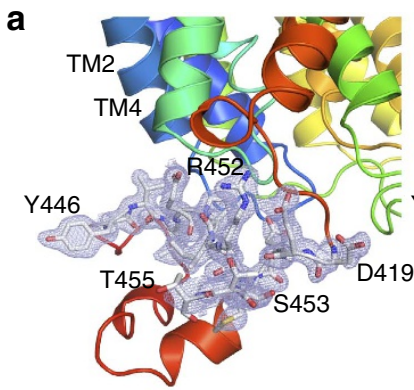

b

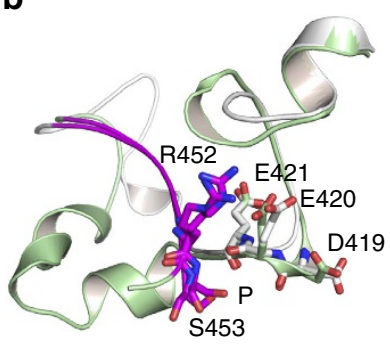

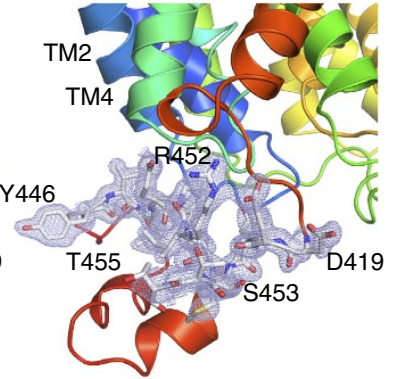

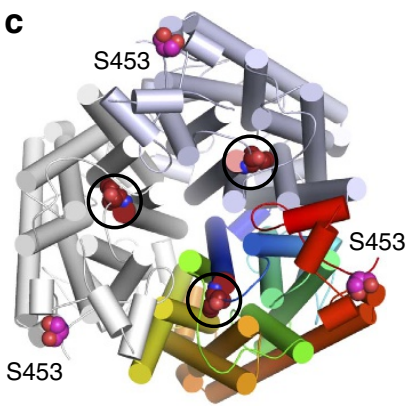

Figure 6 | The Npr1 kinase target Ser453 is dephosphorylated and located in an electronegative pocket. (a) Stereoviews of CaMep2 showing $2 \mathrm{~F}_{\mathrm{o}}-\mathrm{F}_{\mathrm{c}}$ electron density (contoured at $1.0 \sigma$ ) for CTR residues Asp419Met422 and for Tyr446-Thr455 of the Al region. For clarity, the residues shown are coloured white, with oxygen atoms in red and nitrogen atoms in blue. The phosphorylation target residue Ser453 is labelled in bold.

(b) Overlay of the CTRs of ScMep2 (grey) and CaMep2 (green), showing the similar electronegative environment surrounding the phosphorylation site (P). The Al regions are coloured magenta. (c) Cytoplasmic view of the Mep2 trimer indicating the large distance between Ser453 and the channel exits (circles; lle52 lining the channel exit is shown).

channel. The latter model would fit well with the $\mathrm{NH}_{3} / \mathrm{H}^{+}$ symport model in which the proton is relayed by the twin-His motif ${ }^{14}$. The importance of the Tyr-His hydrogen bond is underscored by the fact that its removal in the ScMep2 Y53A mutant results in a constitutively active transporter (Fig. 3).

Phosphorylation causes a conformational change in the CTR. Do the Mep2 structures provide any clues regarding the potential effect of phosphorylation? The side-chain hydroxyl of Ser457/453 is located in a well-defined electronegative pocket that is solvent accessible (Fig. 6). The closest atoms to the serine hydroxyl group are the backbone carbonyl atoms of Asp419, Glu420 and Glu421, which are $3-4 \AA$ away. We therefore predict that phosphorylation of Ser453 will result in steric clashes as well as electrostatic repulsion, which in turn might cause substantial conformational changes within the CTR. To test this hypothesis, we determined the structure of the phosphorylation-mimicking R452D/S453D protein (hereafter termed 'DD mutant'), using data to a resolution of $2.4 \AA$. The additional mutation of the arginine preceding the phosphorylation site was introduced (i) to increase the negative charge density and make it more comparable to a phosphate at neutral $\mathrm{pH}$, and (ii) to further destabilize the interactions of the AI region with the main body of the transporter (Fig. 6). The ammonium uptake activity of the S. cerevisiae version of the DD mutant is the same as that of WT Mep2 and the S453D mutant, indicating that the mutations do not affect transporter functionality in the triple mep $\Delta$ background (Fig. 3). Unexpectedly, the AI segment containing the mutated residues has only undergone a slight shift compared with the WT protein (Fig. 8
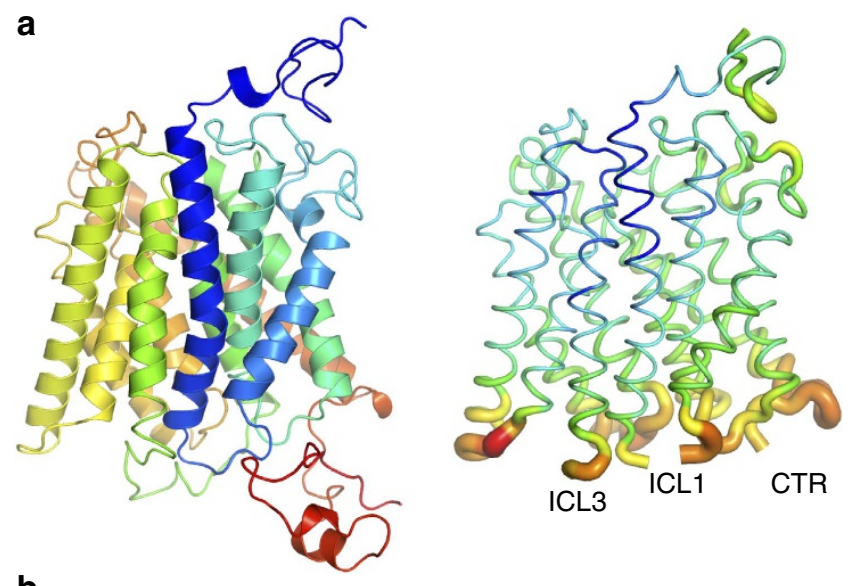

b
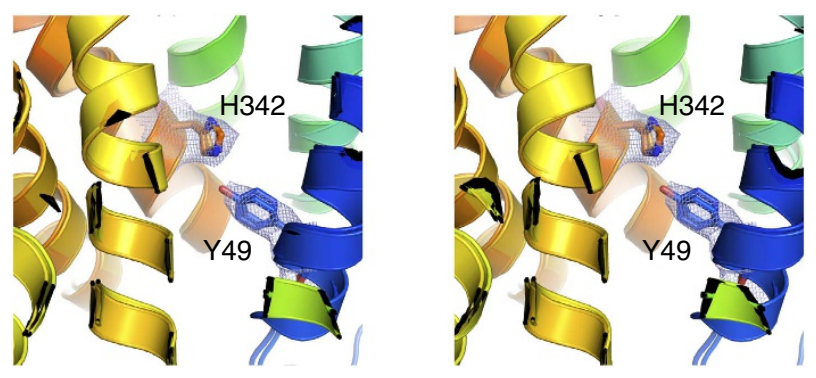

Figure 7 | Effect of removal of the Al region on Mep2 structure. (a) Side views for WT CaMep2 (left) and the truncation mutant $442 \Delta$ (right). The latter is shown as a putty model according to B-factors to illustrate the disorder in the protein on the cytoplasmic side. Missing regions are labelled. (b) Stereo superpositions of WT CaMep2 and the truncation mutant. $2 \mathrm{~F}_{\mathrm{o}}-\mathrm{F}_{\mathrm{c}}$ electron density (contoured at $1.0 \sigma$ ) for residues Tyr49 and His342 is shown for the truncation mutant.
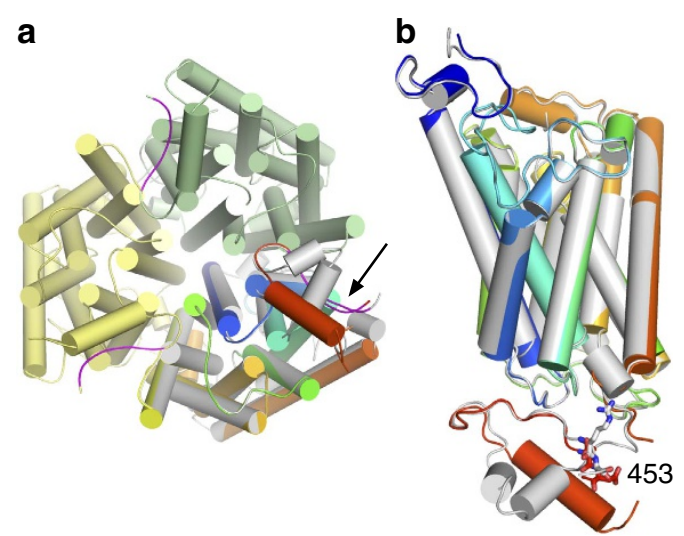

Figure 8 | Phosphorylation causes conformational changes in the CTR. (a) Cytoplasmic view of the DD mutant trimer, with WT CaMep2 superposed in grey for one of the monomers. The arrow indicates the phosphorylation site. The Al region is coloured magenta. (b) Monomer side-view superposition of WT CaMep2 and the DD mutant, showing the conformational change and disorder around the ExxGxD motif. Side chains for residues 452 and 453 are shown as stick models.

and Supplementary Fig. 3). By contrast, the conserved part of the CTR has undergone a large conformational change involving formation of a 12-residue-long $\alpha$-helix from Leu427 to Asp438. In addition, residues Glu420-Leu423 including Glu421 of the ExxGxD motif are now disordered (Fig. 8 and Supplementary Fig. 3). Overall, $\sim 20$ residues are affected by the introduced mutations. This is the first time a large conformational change 
has been observed in an ammonium transporter as a result of a mutation, and confirms previous hypotheses that phosphorylation causes structural changes in the $\mathrm{CTR}^{29,30,33}$. To exclude the possibility that the additional R452D mutation is responsible for the observed changes, we also determined the structure of the 'single D' S453D mutant. As shown in Supplementary Fig. 4, the consequence of the single $\mathrm{D}$ mutation is very similar to that of the DD substitution, with conformational changes and increased dynamics confined to the conserved part of the CTR (Supplementary Fig. 4). To supplement the crystal structures, we also performed modelling and MD studies of WT CaMep2, the DD mutant and phosphorylated protein (S453J). In the WT structure, the acidic residues Asp419, Glu420 and Glu421 are within hydrogen bonding distance of Ser453. After $200 \mathrm{~ns}$ of MD simulation, the interactions between these residues and Ser453 remain intact. The protein backbone has an average r.m.s.d. of only $\sim 3 \AA$ during the 200 -ns simulation, indicating that the protein is stable. There is flexibility in the side chains of the acidic residues so that they are able to form stable hydrogen bonds with Ser453. In particular, persistent hydrogen bonds are observed between the Ser453 hydroxyl group and the acidic group of Glu420, and also between the amine group of Ser453 and the backbone carbonyl of Glu420 (Supplementary Fig. 5). The DD mutant is also stable during the simulations, but the average backbone r.m.s.d of $\sim 3.6 \AA$ suggests slightly more conformational flexibility than WT. As the simulation proceeds, the side chains of the acidic residues move away from Asp452 and Asp453, presumably to avoid electrostatic repulsion. For example, the distance between the Asp453 acidic oxygens and the Glu420 acidic oxygens increases from $\sim 7$ to $>22 \AA$ after $200 \mathrm{~ns}$ simulations, and thus these residues are not interacting. The protein is structurally stable throughout the simulation with little deviation in the other parts of the protein. Finally, the S453J mutant is also stable throughout the 200-ns simulation and has an average backbone deviation of $\sim 3.8 \AA$, which is similar to the DD mutant. The movement of the acidic residues away from Arg452 and Sep453 is more pronounced in this simulation in comparison with the movement away from Asp452 and Asp453 in the DD mutant. The distance between the phosphate of Sep453 and the acidic oxygen atoms of Glu420 is initially $\sim 11 \AA$, but increases to $>30 \AA$ after $200 \mathrm{~ns}$. The short helix formed by residues Leu427 to Asp438 unravels during the simulations to a disordered state. The remainder of the protein is not affected (Supplementary Fig. 5). Thus, the MD simulations support the notion from the crystal structures that phosphorylation generates conformational changes in the conserved part of the CTR. However, the conformational changes for the phosphomimetic mutants in the crystals are confined to the CTR (Fig. 8), and the channels are still closed (Supplementary Fig. 2). One possible explanation is that the mutants do not accurately mimic a phosphoserine, but the observation that the S453D and DD mutants are fully active in the absence of Nprl suggests that the mutations do mimic the effect of phosphorylation (Fig. 3). The fact that the S453D structure was obtained in the presence of $10 \mathrm{mM}$ ammonium ions suggests that the crystallization process favours closed states of the Mep2 channels.

\section{Discussion}

Knowledge about ammonium transporter structure has been obtained from experimental and theoretical studies on bacterial family members. In addition, a number of biochemical and genetic studies are available for bacterial, fungal and plant proteins. These efforts have advanced our knowledge considerably but have not yet yielded atomic-level answers to several important mechanistic questions, including how ammonium transport is regulated in eukaryotes and the mechanism of ammonium signalling. In Arabidopsis thaliana Amt-1;1, phosphorylation of the CTR residue T460 under conditions of high ammonium inhibits transport activity, that is, the default (non-phosphorylated) state of the plant transporter is open. Interestingly, phosphomimetic mutations introduced into one monomer inactivate the entire trimer, indicating that (i) heterotrimerization occurs and (ii) the CTR mediates allosteric regulation of ammonium transport activity via phosphorylation $^{33,34}$. Owing to the lack of structural information for plant AMTs, the details of channel closure and inter-monomer crosstalk are not yet clear. Contrasting with the plant transporters, the inactive states of Mep2 proteins under conditions of high ammonium are non-phosphorylated, with channels that are closed on the cytoplasmic side. The reason why similar transporters such as $A$. thaliana Amt-1;1 and Mep2 are regulated in opposite ways by phosphorylation (inactivation in plants and activation in fungi) is not known. In fungi, preventing ammonium entry via channel closure in ammonium transporters would be one way to alleviate ammonium toxicity, in addition to ammonium excretion via Ato transporters and amino-acid secretion $^{35}$.

By determining the first structures of closed ammonium transporters and comparing those structures with the permanently open bacterial proteins, we demonstrate that Mep2 channel closure is likely due to movements of the CTR and ICL1 and ICL3. More specifically, the close interactions between the CTR and ICL1/ICL3 present in open transporters are disrupted, causing ICL3 to move outwards and block the channel (Figs 4 and 9a). In addition, ICL1 has shifted inwards to contribute to the channel closure by engaging His2 from the twin-His motif via hydrogen bonding with a highly conserved tyrosine hydroxyl group. Upon phosphorylation by the Npr1 kinase in response to nitrogen limitation, the region around the conserved ExxGxD motif undergoes a conformational change that opens the channel (Fig. 9). Importantly, the structural similarities in the TM parts of Mep2 and AfAmt-1 (Fig. 5a) suggest that channel opening/closure does not require substantial changes in the residues lining the channel. How exactly the channel opens and whether opening is intra-monomeric are still open questions; it is possible that the change in the CTR may disrupt its interactions with ICL3 of the neighbouring monomer (Fig. 9b), which could result in opening of the neighbouring channel via inward movement of its ICL3. Owing to the crosstalk between monomers ${ }^{33,34}$, a single phosphorylation event might lead to opening of the entire trimer, although this has not yet been tested (Fig. 9b). Whether or not Mep2 channel opening requires, in addition to phosphorylation, disruption of the Tyr-His2 interaction by the ammonium substrate is not yet clear.

Is our model for opening and closing of Mep2 channels valid for other eukaryotic ammonium transporters? Our structural data support previous studies ${ }^{30-34}$ and clarify the central role of the CTR and cytoplasmic loops in the transition between closed and open states. However, even the otherwise highly similar Mep2 proteins of $S$. cerevisiae and $C$. albicans have different structures for their CTRs (Fig. 1 and Supplementary Fig. 6). In addition, the AI region of the CTR containing the Npr1 kinase site is conserved in only a subset of fungal transporters, suggesting that the details of the structural changes underpinning regulation vary. Nevertheless, given the central role of absolutely conserved residues within the ICL1-ICL3-CTR interaction network (Fig. 4), we propose that the structural basics of fungal ammonium transporter activation are conserved. The fact that Mep2 orthologues of distantly related fungi are fully functional in ammonium transport and signalling in S. cerevisiae supports this notion $^{36-38}$. It should also be noted that the tyrosine residue 
a

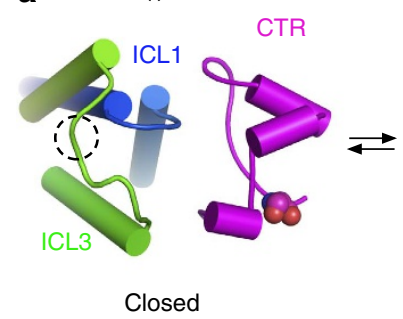

(ii)

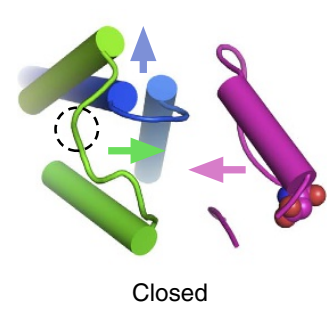

(iii)

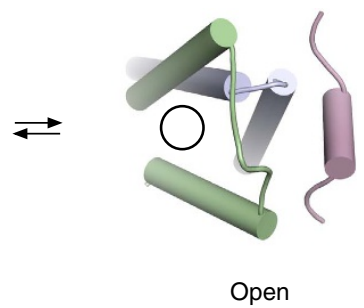

b

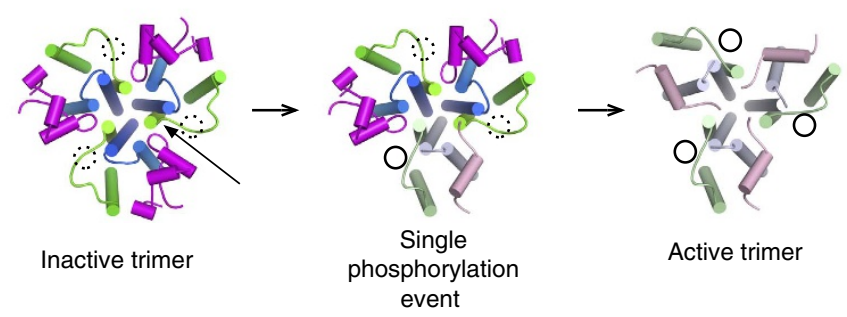

Figure 9 | Schematic model for phosphorylation-based regulation of Mep2 ammonium transporters. (a) In the closed, non-phosphorylated state (i), the CTR (magenta) and ICL3 (green) are far apart with the latter blocking the intracellular channel exit (indicated with a hatched circle). Upon phosphorylation and mimicked by the CaMep2 S453D and DD mutants (ii), the region around the ExxGxD motif undergoes a conformational change that results in the CTR interacting with the inward-moving ICL3, opening the channel (full circle) (iii). The arrows depict the movements of important structural elements. The open-channel Mep2 structure is represented by archaebacterial Amt-1 and shown in lighter colours consistent with Fig. 4. As discussed in the text, similar structural arrangements may occur in plant AMTs. In this case however, the open channel corresponds to the non-phosphorylated state; phosphorylation breaks the CTR-ICL3 interactions leading to channel closure. (b) Model based on AMT transporter analogy ${ }^{29,33}$ showing how phosphorylation of a Mep2 monomer might allosterically open channels in the entire trimer via disruption of the interactions between the CTR and ICL3 of a neighbouring monomer (arrow).

interacting with His2 is highly conserved in fungal Mep2 orthologues, suggesting that the Tyr-His2 hydrogen bond might be a general way to close Mep2 proteins.

With regards to plant AMTs, it has been proposed that phosphorylation at T460 generates conformational changes that would close the neighbouring pore via the $\mathrm{C}$ terminus. This assumption was based partly on a homology model for Amt-1;1 based on the (open) archaebacterial AfAmt-1 structure, which suggested that the $C$ terminus of Amt-1;1 would extend further to the neighbouring monomer ${ }^{33}$. Our Mep2 structures show that this assumption may not be correct (Fig. 4 and Supplementary Fig. 6). In addition, the considerable differences between structurally resolved CTR domains means that the exact environment of T460 in Amt-1;1 is also not known (Supplementary Fig. 6). Based on the available structural information, we consider it more likely that phosphorylation-mediated pore closure in Amt-1;1 is intra-monomeric, via disruption of the interactions between the CTR and ICL1/ICL3 (for example, Y467-H239 and D458-K71) ${ }^{33}$. There is generally no equivalent for CaMep2 Tyr49 in plant AMTs, indicating that a Tyr-His2 hydrogen bond as observed in Mep2 may not contribute to the closed state in plant transporters.

We propose that intra-monomeric CTR-ICL1/ICL3 interactions lie at the basis of regulation of both fungal and plant ammonium transporters; close interactions generate open channels, whereas the lack of 'intra-' interactions leads to inactive states. The need to regulate in opposite ways may be the reason why the phosphorylation sites are in different parts of the CTR, that is, centrally located close to the ExxGxD motif in AMTs and peripherally in Mep2. In this way, phosphorylation can either lead to channel closing (in the case of AMTs) or channel opening in the case of Mep2. Our model also provides an explanation for the observation that certain mutations within the CTR completely abolish transport activity. An example of an inactivating residue is the glycine of the ExxGxD motif of the CTR. Mutation of this residue (G393 in EcAmtB; G456 in AtAmt-1;1) inactivates transporters as diverse as Escherichia coli AmtB and A. thaliana Amt-1;1 (refs 32,33). Such mutations likely cause structural changes in the CTR that prevent close contacts between the CTR and ICL1/ICL3, thereby stabilizing a closed state that may be similar to that observed in Mep2.

Regulation and modulation of membrane transport by phosphorylation is known to occur in, for example, aquaporins ${ }^{39}$ and urea transporters ${ }^{40}$, and is likely to be a common theme for eukaryotic channels and transporters. Recently, phosphorylation was also shown to modulate substrate affinity in nitrate transporters $^{41}$. With respect to ammonium transport, phosphorylation has thus far only been shown for A. thaliana AMTs and for S. cerevisiae Mep2 (refs 29,30,32,33). However, the absence of $\mathrm{GlnK}$ proteins in eukaryotes suggests that phosphorylation-based regulation of ammonium transport may be widespread. Nevertheless, as discussed above, considerable differences may exist between different species.

With respect to Mep2-mediated signalling to induce pseudohyphal growth, two models have been put forward as to how this occurs and why it is specific to Mep2 proteins. In one model, signalling is proposed to depend on the nature of the transported substrate, which might be different in certain subfamilies of ammonium transporters (for example, Mep1/Mep3 versus Mep2). For example, $\mathrm{NH}_{3}$ uniport or symport of $\mathrm{NH}_{3} / \mathrm{H}^{+}$ might result in changes in local $\mathrm{pH}$, but $\mathrm{NH}_{4}^{+}$uniport might not $^{10,21}$, and this difference might determine signalling. In the other model, signalling is thought to require a distinct conformation of the Mep2 transporter occurring during the transport cycle ${ }^{8}$. While the current study does not specifically address the mechanism of signalling underlying pseudohyphal growth, our structures do show that Mep2 proteins can assume different conformations.

It is clear that ammonium transport across biomembranes remains a fascinating and challenging field in large part due to the 
unique properties of the substrate. Our Mep2 structural work now provides a foundation for future studies to uncover the details of the structural changes that occur during eukaryotic ammonium transport and signaling, and to assess the possibility to utilize small molecules to shut down ammonium sensing and downstream signalling pathways in pathogenic fungi.

\section{Methods}

Mep2 overexpression and purification. Ammonium transporter genes were amplified from genomic DNA or cDNA by PCR (Phusion, New England Biolabs). In both ScMEP2 and CaMEP2, Asn 4 was replaced by a glutamine to prevent glycosylation. In order to allow transformation of yeast by recombination, the following primer extensions were used: forward $5^{\prime}$-GAAAAAACCCCGGA TTCTAGAACTAGTGGATCCTCC- ${ }^{\prime}$ and reverse $5^{\prime}$-TGACTCGAGTTATGCA CCGTGGTGGTGATGGTGATG-3' ${ }^{\prime}$. These primers result in a construct that lacks the cleavable $\mathrm{N}$ - and $\mathrm{C}$-terminal tags present in the original vector ${ }^{42}$, and replaces these with a C-terminal hexa-histidine tag. Recombination in yeast strain W303 pep4 4 was carried out using $\sim 50-100 \mathrm{ng}$ of SmaI-digested vector $83 \mathrm{v} \Delta$ (ref. 42) and at least a fourfold molar excess of PCR product via the lithium acetate method. Transformants were selected on SCD -His plates incubated at $30^{\circ} \mathrm{C}$. Construction of mutant CaMEP2 genes was done using the Q5 site-directed mutagenesis kit (NEB) per manufacturer's instructions. Three CaMep2 mutants were made for crystallization: the first mutant is a C-terminal truncation mutant $442 \Delta$, lacking residues $443-480$ including the AI domain. The second mutant, $\mathrm{R} 452 \mathrm{D} / \mathrm{S} 453 \mathrm{D}$, mimics the protein phosphorylated at Ser453. Given that phosphate is predominantly charged -2 at physiological $\mathrm{pH}$, we introduced the second aspartate residue for Arg452. However, we also constructed the 'single D', S453D CaMep2 variant.

For expression, cells were grown in shaker flasks at $30^{\circ} \mathrm{C}$ for $\sim 24 \mathrm{~h}$ in synthetic minimal medium lacking histidine and with $1 \%(\mathrm{w} / \mathrm{v})$ glucose to a typical $\mathrm{OD}_{600}$ of 6-8. Cells were subsequently spun down for $15 \mathrm{~min}$ at $4,000 \mathrm{~g}$ and resuspended in YP medium containing 1.5\% (w/v) galactose, followed by another $16-20 \mathrm{~h}$ growth at $30^{\circ} \mathrm{C} / 160$ r.p.m. and harvesting by centrifugation. Final $\mathrm{OD}_{600}$ values typically reached $18-20$. Cells were lysed by bead beating (Biospec) for $5 \times 1 \mathrm{~min}$ with $1 \mathrm{~min}$ intervals on ice, or by 1-2 passes through a cell disrupter operated at 35,000 p.s.i. (TS-Series $0.75 \mathrm{~kW}$; Constant Systems). Membranes were collected from the suspension by centrifugation at $200,000 \mathrm{~g}$ for $90 \mathrm{~min}$ (45Ti rotor; Beckmann Coulter). Membrane protein extraction was performed by homogenization in a $1: 1(\mathrm{w} / \mathrm{w})$ mixture of dodecyl- $\beta$-D-maltoside and decyl- $\beta$-D-maltoside (DDM/DM) followed by stirring at $4{ }^{\circ} \mathrm{C}$ overnight. Typically, $1 \mathrm{~g}(1 \% \mathrm{w} / \mathrm{v})$ of total detergent was used for membranes from 21 of cells. The membrane extract was centrifuged for $35 \mathrm{~min}$ at $200,000 \mathrm{~g}$ and the supernatant was loaded onto a $10-\mathrm{ml}$ Nickel column (Chelating Sepharose; GE Healthcare) equilibrated in $20 \mathrm{mM}$ Tris $/ 300 \mathrm{mM} \mathrm{NaCl} / 0.2 \% \mathrm{DDM}, \mathrm{pH}$. The column was washed with 15 column volumes buffer containing $30 \mathrm{mM}$ imidazole and eluted in 3 column volumes with $250 \mathrm{mM}$ imidazole. Proteins were purified to homogeneity by gel filtration chromatography in $10 \mathrm{mM} \mathrm{HEPES} / 100 \mathrm{mM}$ $\mathrm{NaCl} / 0.05 \%$ DDM, pH 7-7.5. For polishing and detergent exchange, a second gel filtration column was performed using various detergents. In the case of ScMep2, diffracting crystals were obtained only with $0.05 \%$ decyl-maltose neopentyl glycol. For the more stable CaMep2 protein, we obtained crystals in, for example, nonyl-glucoside, decyl-maltoside and octyl-glucose neopentyl glycol. Proteins were concentrated to $7-15 \mathrm{mg} \mathrm{ml}^{-1}$ using $100 \mathrm{kDa}$ cutoff centrifugal devices (Millipore), flash-frozen and stored at $-80{ }^{\circ} \mathrm{C}$ before use.

Crystallization and structure determination. Crystallization screening trials by sitting drop vapour diffusion were set up at 4 and $20^{\circ} \mathrm{C}$ using in-house screens and the MemGold 1 and 2 screens (Molecular Dimensions) with a Mosquito crystallization robot. Crystals were harvested directly from the initial trials or optimized by sitting or hanging drop vapour diffusion using larger drops (typically $2-3 \mu \mathrm{l}$ total volume). Bar-shaped crystals for ScMep2 diffracting to $3.2 \AA$ resolution were obtained from $50 \mathrm{mM}$ 2-(N-morpholino)ethanesulfonic acid (MES)/0.2 M di-ammonium hydrogen phosphate/30\% PEG 400, pH 6 . They belong to space group $\mathrm{P} 2{ }_{1} 2_{1} 2_{1}$ and have nine molecules (three trimers) in the asymmetric unit (AU). Well-diffracting crystals for CaMep2 were obtained in space group P3 from $0.1 \mathrm{M}$ MES/0.2 M lithium sulphate/20\% PEG400, pH 6 (two molecules per AU). An additional crystal form in space group R3 was grown in $0.04 \mathrm{M}$ Tris/0.04 M NaCl/ 27\% PEG350 MME, pH 8 (one molecule per AU). Diffracting crystals for the phosporylation-mimicking CaMep2 DD mutant were obtained in space group $\mathrm{P}_{3} 22$ from $0.1 \mathrm{M}$ sodium acetate/15-20\% PEG400, pH 5 (using decyl-maltoside as detergent; one molecule per AU), while S453D mutant crystals grew in 24\% PEG400/ $0.05 \mathrm{M}$ sodium acetate, $\mathrm{pH} 5.4 / 0.05 \mathrm{M}$ magnesium acetate tetrahydrate $/ 10 \mathrm{mM}$ $\mathrm{NH}_{4} \mathrm{Cl}$ (space group $\mathrm{R}_{2}$; one molecule per AU). Finally, the $442 \Delta$ truncation mutant gave crystals under many different conditions, but most of these diffracted poorly or not at all. A reasonable low-resolution data set (3.4 $\AA$ resolution) was eventually obtained from a crystal grown in $24 \%$ PEG400/0.05 M sodium acetate/ $0.05 \mathrm{M}$ magnesium acetate, $\mathrm{pH} 6.1$ (space group $\mathrm{R} 3_{2}$ ). Diffraction data were collected at the Diamond Light Source and processed with XDS ${ }^{43}$ or HKL2000 (ref. 44).
For MR, a search model was constructed with Sculptor within Phenix ${ }^{45}$, using a sequence alignment of ScMep2 with Archaeoglobus fulgidus Amt-1 (PDB ID 2B2H; $\sim 40 \%$ sequence identity to ScMep2). A clear solution with nine molecules (three trimers) in the AU was obtained using Phaser ${ }^{46}$. The model was subsequently completed by iterative rounds of manual building within Coot $^{47}$ followed by refinement within Phenix. The structures for WT CaMep2 were solved using the best-defined monomer of ScMep2 (60\% sequence identity with CaMep2) in MR with Phaser, followed by automated model building within Phenix. Finally, the structures of the three mutant CaMep2 proteins were solved using WT CaMep2 as the search model. The data collection and refinement statistics for all six solved structures have been summarized in Supplementary Tables 1 and 2.

Growth assays. The $S$. cerevisiae haploid triple mep $\Delta$ strain $(\Sigma 1278 \mathrm{~b}$ MAT $\alpha$ mep1::LEU2 mep2::LEU2 mep3::G418 ura3-52) and triple mep $\Delta$ npr1 $\Delta$ strain ( $\Sigma 1278$ b MAT $\alpha$ mep1::LEU2 mep2::LEU2 mep3::G418 npr1::NAT1 ura3-52) were generated by integrating the NAT1 resistance gene at one NPR1 locus in the diploid strain MLY131 (ref. 6), followed by isolation of individual haploid strains Cells were grown in synthetic minimal medium with glucose $(2 \%)$ as the carbon source and ammonium sulphate $(1 \mathrm{mM})$ or glutamate $(0.1 \%)$ as the nitrogen source. Yeast cells were transformed as described ${ }^{48}$. All DNA sequences encoding epitope-tagged ScMep2 and its mutant derivatives were generated by PCR and homologous recombination using the vector pRS316 (ref. 49). In each case, the ScMEP2 sequences included the ScMEP2 promoter $(1 \mathrm{~kb})$, the ScMEP2 terminator and sequences coding for a His- 6 epitope at the C-terminal end of the protein. All Mep2-His fusions contain the N4Q mutation to prevent glycosylation of Mep2 (ref. 50). All newly generated plasmid inserts were verified by DNA sequencing. For growth assays, S. cerevisiae cells containing plasmids expressing ScMep2 or mutant derivatives were grown overnight in synthetic minimal glutamate medium, washed, spotted by robot onto solid agar plates and culture growth followed by time course photography. Images were then processed to quantify the growth of each strain over 3 days as described ${ }^{51}$

Protein modelling. The MODELLER (version 9.15) software package ${ }^{52}$ was used to build protein structures for MD simulations. This method was required to construct two complete protein models, the double mutant R452D/S453D (with the four missing residues from the X-ray structure added) and also the construct in which the mutation at position 452 is reverted to R, and D453 is replaced with a phosphoserine. The quality of these models was assessed using normalized Discrete Optimized Protein Energy (DOPE) values and the molpdf assessment function within the MODELLER package. The model R452D/S453D mutant has a molpdf assessment score of 1854.05, and a DOPE assessment score of -60920.55 . The model of the S453J mutant has a molpdf assessment score of 1857.01 and a DOPE assessment score of -61032.15 .

MD simulations. WT and model structures were embedded into a pre-equilibrated lipid bilayer composed of 512 dipalmitoylphosphatidylcholine lipids using the InflateGRO2 computer programme ${ }^{53}$. The bilayers were then solvated with the SPC water model ${ }^{54}$ and counterions were added to achieve a charge neutral state. All simulations were performed with the GROMACS package (version 4.5 .5$)^{55}$, and the GROMOS96 43alp force field. During simulation time, the temperature was maintained at $310 \mathrm{~K}$ using the Nosé-Hoover thermostat ${ }^{56,57}$ with a coupling constant of 0.5 ps. Pressure was maintained at 1 bar using semi-isotropic coupling with the Parrinello-Rahman barostat ${ }^{58}$ and a time constant of 5 ps. Electrostatic interactions were treated using the smooth particle mesh Ewald algorithm ${ }^{59}$ with a short-range cutoff of $0.9 \mathrm{~nm}$. Van der Waals interactions were truncated at $1.4 \mathrm{~nm}$ with a long-range dispersion correction applied to energy and pressure. The neighbour list was updated every five steps. All bonds were constrained with the LINCS algorithm ${ }^{60}$, so that a 2 -fs time step could be applied throughout. The phospholipid parameters for the dipalmitoylphosphatidylcholine lipids were based on the work of Berger ${ }^{61}$. The embedded proteins were simulated for $200 \mathrm{~ns}$ each; a repeat simulation was performed for each system with different initial velocities to ensure reproducibility. To keep the c.p.u. times within reasonable limits, all simulations were performed on Mep2 monomers. This is also consistent with previous simulations for $E$. coli $\mathrm{AmtB}^{16-18}$.

\section{References}

1. Holsbeeks, I., Lagatie, O., Van Nuland, A., Van de Velde, S. \& Thevelein, J. M. The eukaryotic plasma membrane as a nutrient-sensing device. Trends Biochem. Sci. 29, 556-564 (2004).

2. Conrad, M. et al. Nutrient sensing and signaling in the yeast Saccharomyces cerevisiae. FEMS Microbiol. Rev. 38, 254-299 (2014).

3. Saier, M. H., Reddy, V. S., Tamang, D. G. \& Vastermark, A. The transporter classification database. Nucleic Acids Res. 42, D251-D258 (2014).

4. Marini, A. M., Soussi-Boudekou, S., Vissers, S. \& Andre, B. A family of ammonium transporters in Saccharomyces cerevisiae. Mol. Cell Biol. 17, 4282-4293 (1997).

5. Van Zeebroeck, G., Kimpe, M., Vandormael, P. \& Thevelein, J. M. A splitubiquitin two-hybrid screen for proteins physically interacting with the yeast 
amino acid transceptor Gap1 and ammonium transceptor Mep2. PLoS ONE 6, e24275 (2011).

6. Lorenz, M. C. \& Heitman, J. The MEP2 ammonium permease regulates pseudohyphal differentiation in Saccharomyces cerevisiae. EMBO J. 17, 1236-1247 (1998).

7. Shnaiderman, C., Miyara, I., Kobiler, I., Sherman, A. \& Prusky, D. Differential activation of ammonium transporters during the accumulation of ammonia by Colletotrichum gloeosporioides and its effect on appressoria formation andpathogenicity. Mol. Plant Microbe Interact. 26, 345-355 (2013).

8. Rutherford, J. C., Chua, G., Hughes, T., Cardenas, M. E. \& Heitman, J. A Mep2dependent transcriptional profile links permease function to gene expression during pseudohyphal growth in Saccharomyces cerevisiae. Mol. Biol. Cell 19, 3028-3039.

9. Biswas, K. \& Morschhäuser, J. The Mep2p ammonium permease controls nitrogen starvation-induced filamentous growth in Candida albicans. Mol. Microbiol. 56, 649-669 (2005).

10. Boeckstaens, M., André, B. \& Marini, A. M. Distinct transport mechanisms in yeast ammonium transport/sensor proteins of the Mep/Amt/Rh family and impact on filamentation. J. Biol. Chem. 283, 21362-21370 (2008).

11. Boeckstaens, M., André, B. \& Marini, A. M. The yeast ammonium transport protein Mep2 and its positive regulator, the Npr1 kinase, play an important role in normal and pseudohyphal growth on various nitrogen media through retrieval of excreted ammonium. Mol. Microbiol. 64, 534-546 (2007).

12. Gruswitz, F. et al. Function of human Rh based on structure of RhCG at 2.1A. Proc. Natl Acad. Sci. USA 107, 9638-9643 (2010).

13. Khademi, S. et al. Mechanism of ammonia transport by Amt/MEP/Rh: structure of AmtB at 1.35A. Science 305, 1587-1594 (2004).

14. Andrade, S. L., Dickmanns, A., Ficner, R. \& Einsle, O. Crystal structure of the archaeal ammonium transporter Amt-1 from Archaeoglobus fulgidus. Proc. Natl Acad. Sci. USA 102, 14994-14999 (2005).

15. Javelle, A. et al. An unusual twin-his arrangement in the pore of ammonia channels is essential for substrate conductance. J. Biol. Chem. 281, 39492-39498 (2006).

16. Wang, S., Orabi, E. A., Baday, S., Bernèche, S. \& Lamoureux, G. Ammonium transporters achieve charge transfer by fragmenting their substrate. J. Am. Chem. Soc. 134, 10419-10427 (2012).

17. Wang, J. et al. Ammonium transport proteins with changes in one of the conserved pore histidines have different performance in ammonia and methylamine conduction. PLoS ONE 8, e62745 (2013).

18. Lin, Y., Cao, Z. \& Mo, Y. Molecular dynamics simulations on the Escherichia coli ammonia channel protein AmtB: mechanism of ammonia/ammonium transport. J. Am. Chem. Soc. 128, 10876-10884 (2006).

19. Akgun, U. \& Khademi, S. Periplasmic vestibule plays an important role for solute recruitment, selectivity, and gating in the $\mathrm{Rh} / \mathrm{Amt} / \mathrm{MEP}$ superfamily. Proc. Natl Acad. Sci. USA 108, 3970-3975 (2011).

20. Zheng, L., Kostrewa, D., Bernèche, S., Winkler, F. K. \& Li, X. D. The mechanism of ammonia transport based on the crystal structure of AmtB of Escherichia coli. Proc. Natl Acad. Sci. USA 101, 17090-17095 (2004)

21. Baday, S., Wang, S., Lamoureux, G. \& Bernèche, S. Different hydration patterns in the pores of $\mathrm{AmtB}$ and $\mathrm{RhCG}$ could determine their transport mechanisms. Biochemistry 52, 7091-7098 (2013).

22. van Heeswijk, W. C., Westerhoff, H. V. \& Boogerd, F. C. Nitrogen assimilation in Escherichia coli: putting molecular data into a systems perspective. Microbiol. Mol. Biol. Rev. 77, 628-695 (2013).

23. Wacker, T., Garcia-Celma, J. J., Lewe, P. \& Andrade, S. L. Direct observation of electrogenic $\mathrm{NH} 4(+)$ transport in ammonium transport (Amt) proteins. Proc. Natl Acad. Sci. USA 111, 9995-10000 (2014).

24. Neuhäuser, B. \& Ludewig, U. Uncoupling of ionic currents from substrate transport in the plant ammonium transporter AtAMT1;2. J. Biol. Chem. 289, 11650-11655 (2014).

25. Monfort, P., Kosenko, E., Erceg, S., Canales, J. J. \& Felipo, V. Molecular mechanism of acute ammonia toxicity: role of NMDA receptors. Neurochem. Int. 41, 95-102 (2002).

26. Thomas, G., Coutts, G. \& Merrick, M. The glnKamtB operon. A conserved gene pair in prokaryotes. Trends Genet. 16, 11-14 (2000).

27. Gruswitz, F., O'Connell, III J. \& Stroud, R. M. Inhibitory complex of the transmembrane ammonia channel, $\mathrm{AmtB}$, and the cytosolic regulatory protein, GlnK, at 1.96A. Proc. Natl Acad. Sci. USA 104, 42-47 (2006).

28. Durand, A. \& Merrick, M. In vitro analysis of the Escherichia coli AmtB-GlnK complex reveals a stoichiometric interaction and sensitivity to ATP and 2oxoglutarate. J. Biol. Chem. 281, 29558-29567 (2006).

29. Lanquar, V. \& Frommer, W. B. Adjusting ammonium uptake via phosphorylation. Plant Signal. Behav. 5, 736-738 (2010).

30. Boeckstaens, M., Llinares, E., Van Vooren, P. \& Marini, A. M. The TORC1 effector kinase Npr1 fine tunes the inherent activity of the Mep2 ammonium transport protein. Nat. Commun. 5, 3101-3110 (2014).
31. Graff, L. et al. N- terminal cysteines affect oligomer stability of the allosterically regulated ammonium transporter LeAMT1;1. J. Exp. Bot. 62, 1361-1373 (2011).

32. Severi, E., Javelle, A. \& Merrick, M. The conserved carboxy-terminal region of the ammonia channel AmtB plays a critical role in channel function. Mol. Membr. Biol. 24, 161-171 (2007).

33. Loqué, D., Lalonde, S., Looger, L. L., von Wirén, N. \& Frommer, W. B. A cytosolic trans-activation domain essential for ammonium uptake. Nature 446, 195-198 (2007).

34. Yuan, L. et al. Allosteric regulation of transport activity by heterotrimerization of Arabidopsis ammonium transporter complexes in vivo. Plant Cell 25, 974-984 (2013).

35. Hess, D. C., Lu, W., Rabinowitz, J. D. \& Botstein, D. Ammonium toxicity and potassium limitation in yeast. PLoS Biol. 4, e351 (2006).

36. Smith, D. G., Garcia-Pedrajas, M. D., Gold, S. E. \& Perlin, M. H. Isolation and characterization from pathogenic fungi of genes encoding ammonium permeases and their roles in dimorphism. Mol. Microbiol. 50, 259-275 (2003).

37. Teichert, S., Rutherford, J. C., Wottawa, M., Heitman, J. \& Tudzynski, B. Impact of ammonium permeases mepA, mepB, and mepC on nitrogenregulated secondary metabolism in Fusarium fujikuroi. Eukaryot. Cell 7, 187-201 (2008).

38. Javelle, A. et al. Molecular characterization, function and regulation of ammonium transporters (Amt) and ammonium-metabolizing enzymes (GS, NADP-GDH) in the ectomycorrhizal fungus Hebeloma cylindrosporum. Mol. Microbiol. 47, 411-430 (2003).

39. Törnroth-Horsefield, S., Hedfalk, K., Fischer, G., Lindkvist-Petersson, K. \& Neutze, R. Structural insights into eukaryotic aquaporin regulation. FEBS Lett. 584, 2580-2588 (2006).

40. Klein, J. D. Expression of urea transporters and their regulation. Subcell. Biochem. 73, 79-107 (2014).

41. Parker, J. L. \& Newstead, S. Molecular basis of nitrate uptake by the plant nitrate transporter NRT1.1. Nature 507, 68-72 (2014).

42. Hays, F. A., Roe-Zurz, Z. \& Stroud, R. M. Overexpression and purification of integral membrane proteins in yeast. Methods Enzymol. 470, 695-707 (2010).

43. Kabsch, W. XDS. Acta Crystallogr. D66, 125-132 (2010).

44. Otwinowski, Z. \& Minor, W. Processing of X-ray diffraction data collected in oscillation mode. Methods Enzymol. 276, 307-326 (2003).

45. Afonine, P. V. et al. Towards automated crystallographic structure refinement with phenix.refine. Acta Crystallogr. D68, 352-367 (2012).

46. McCoy, A. J., Grosse-Kunstleve, R. W., Storoni, L. C. \& Read, R. J. Likelihoodenhanced fast translation functions. Acta Crystallogr. D61, 458-464 (2005).

47. Emsley, P. \& Cowtan, K. Coot: model-building tools for molecular graphics. Acta Crystallogr. D60, 2126-2132 (2004).

48. Schiestl, R. H. \& Gietz, R. D. High efficiency transformation of intact yeast cells using single stranded nucleic acids as a carrier. Curr. Genet. 16, 339-346 (1989).

49. Ma, H., Kunes, S., Schatz, P. J. \& Botstein, D. Plasmid construction by homologous recombination in yeast. Gene 58, 201-216 (1987).

50. Marini, A. M. \& André, B. In vivo N-glycosylation of the Mep2 high-affinity ammonium transporter of Saccharomyces cerevisiae reveals an extracytosolic N-terminus. Mol. Microbiol. 38, 552-564 (2000).

51. Addinall, S. G. et al. Quantitative fitness analysis shows that NMD proteins and many other protein complexes suppress or enhance distinct telomere cap defects. PLoS Genet. 7, e1001362 (2011).

52. Marti-Renom, M. A. et al. Comparative protein structure modeling of genes and genomes. Annu. Rev. Biophys. Biomol. Struct. 29, 291-325 (2000).

53. Schmidt, T. H. \& Kandt, C. LAMBADA and InflateGRO2: efficient membrane alignment and insertion of membrane proteins for molecular dynamics simulations. J. Chem. Inf. Model. 52, 2657-2669 (2012).

54. Berendsen, H. J. C., Postma, J. P., van Gunsteren, W. F. \& Hermans, J. Interaction models for water in relation to protein hydration. Intermolecular Forces 331-342 (Springer, 1981).

55. Van Der Spoel, D. et al. GROMACS: fast, flexible, and free. J. Comput. Chem. 26, 1701-1718 (2005).

56. Nosé, S. A unified formulation of the constant temperature molecular dynamics methods. J. Chem. Phys. 81, 511-519 (1984).

57. Hoover, W. G. Canonical dynamics: equilibrium phase-space distributions. Phys. Rev. A 31, 1695 (1985).

58. Parrinello, M. \& Rahman, A. Polymorphic transitions in single crystals: a new molecular dynamics method. J. Appl. Phys. 52, 7182-7190 (1981).

59. Essmann, U. et al. A smooth particle mesh Ewald method. J. Chem. Phys. 103, 8577-8593 (1995).

60. Hess, B., Bekker, H., Berendsen, H. J. \& Fraaije, J. G. LINCS: a linear constraint solver for molecular simulations. J. Comput. Chem. 18, 1463-1472 (1997).

61. Berger, O., Edholm, O. \& Jähnig, F. Molecular dynamics simulations of a fluid bilayer of dipalmitoylphosphatidylcholine at full hydration, constant pressure, and constant temperature. Biophys. J. 72, 2002-2013 (1997).

62. The PyMOL Molecular Graphics System. version 1.7.4 (Schrödinger, LLC). 


\section{Acknowledgements}

B.v.d.B. acknowledges the Royal Society for salary support. We also thank Peter Banks, manager of the high-throughput screening facility at Newcastle University, for advice and assistance. We also acknowledge Siobhan Lister for expression and purification of the CaMep2 S453D mutant. A.C. was supported by a UK Biotechnology and Biological Sciences Research Council studentship. This project made use of time on ARCHER granted via the UK High-End Computing Consortium for Biomolecular Simulation, HECBioSim (http://hecbiosim.ac.uk), supported by EPSRC (grant no. EP/L000253/1).

\section{Author contributions}

B.v.d.B. performed the experiments related to Mep2 structure determination, designed research and wrote the paper. A.C. performed ammonium growth experiments of Mep variants. D.J. and S.K. performed modelling studies and MD simulations. A.B. collected the X-ray synchrotron data and maintained the Newcastle Structural Biology Laboratory. J.C.R. designed research related to the $S$. cerevisiae growth assays.

\section{Additional information}

Accession codes: The atomic coordinates and the associated structure factors have been deposited in the Protein Data Bank (http:// www.pdbe.org) with accession codes 5AEX
(ScMep2), 5AEZ (CaMep2; R3), 5AF1 (CaMep2; P3), 5AID (CaMep2; 442 $)$ ), 5AH3 (CaMep2; R452D/S453D) and 5FUF (CaMep2; S453D).

Supplementary Information accompanies this paper at http://www.nature.com/ naturecommunications

Competing financial interests: The authors declare no competing financial interests.

Reprints and permission information is available online at http://npg.nature.com/ reprintsandpermissions/

How to cite this article: van den Berg, B. et al. Structural basis for Mep2 ammonium transceptor activation by phosphorylation. Nat. Commun. 7:11337 doi: 10.1038/ncomms11337 (2016).

(c) (i) This work is licensed under a Creative Commons Attribution 4.0 International License. The images or other third party material in this article are included in the article's Creative Commons license, unless indicated otherwise in the credit line; if the material is not included under the Creative Commons license, users will need to obtain permission from the license holder to reproduce the material. To view a copy of this license, visit http://creativecommons.org/licenses/by/4.0/ 